\title{
Fatores que Influenciam a Escolha da Especialização Médica pelos Estudantes de Medicina em uma Instituição de Ensino de Curitiba (PR)
}

\author{
Factors that Influence the Choice of Medical \\ Specialty by Medical Students of an Educational \\ Institution in Curitiba (PR)
}

\author{
Jessica Belei Martins \\ Fernanda Pombo Rodriguez ${ }^{I}$ \\ Izabel Cristina Meister Martins Coelho \\ Elisângela de Mattos e Silva ${ }^{I}$
}

\section{PALAVRAS-CHAVE \\ - Escolha da Profissão. \\ - Residência Médica. \\ - Medicina. \\ - Especialização Médica.}

\begin{abstract}
RESUMO
A escolha da especialidade médica é de suma importância para o estudante de Medicina, uma vez que representa a escolha da sua prática diária. Embora a realização acadêmica, considerações financeiras e preferências de estilo de vida influenciem a escolha, os aspectos intrapessoais têm impacto na decisão do médico. Objetivos: Avaliar os principais fatores que influenciam o acadêmico de Medicina na escolha da especialidade médica e identificar as áreas de atuação preferidas pelos estudantes dos quatro primeiros anos de uma instituição de ensino superior de Curitiba (PR). Resultados: Dos 397 estudantes, 307 responderam a um questionário previamente elaborado para este estudo, sendo que 291 foram considerados válidos. Duzentos e quatro estudantes são do sexo feminino e 87 do sexo masculino. Os fatores de influência foram comparados entre os estudantes dos diferentes anos e com resultados publicados na literatura. Os principais fatores considerados pelos estudantes nesta decisão foram: conhecimento mais amplo ou específico, contato com o paciente, local de atuação profissional e estilo de vida após a residência médica. Para as mulheres, a pressão do dia a dia durante o exercício da especialidade, a relação a longo prazo com o paciente e a facilidade de emprego foram significativamente mais relevantes na escolha da especialidade do que para os homens. Pai ou mãe médicos influenciam os estudantes na decisão da escolha da especialidade. O perfil financeiro do estudante influenciou a escolha quando relacionado a retorno financeiro a longo prazo, sendo mais relevante para os estudantes com renda familiar mensal entre 6 mil e 15 mil reais ou maior que 20 mil reais. A especialidade de Cirurgia Geral foi a preferida pelos estudantes, com predominância no sexo masculino, seguida de Pediatria, Clínica Médica e Psiquiatria. Conclusões: Estilo de vida, local de atuação e retorno financeiro precoce foram considerados igualmente importantes por estudantes de todos os anos avaliados. Devido à grande quantidade de variáveis avaliadas e de fatores que influenciam esta decisão, estudos adicionais são necessários para comparações mais significativas com a literatura disponível.
\end{abstract}




\section{KEY-WORDS}

- Career Choice.

- Internship and Residency.

- Medicine.

- Medical Specialty.

\begin{abstract}
Choosing a medical specialty is extremely important for medical students as it represents the choice of their day-to-day practice. While this choice is influenced by academic achievement, financial considerations and lifestyle preferences, intrapersonal aspects also have an impact on the decision. Objectives: To evaluate the main factors that influence medical students in their choice of medical specialty, and to identify the areas of practice preferred by students in the first four years of a Higher Education Institution. Results: Out of a total of 397 students, 307 answered a questionnaire previously prepared for this study, and of these, 291 were considered valid. 204 female, and 87 male. Influencing factors were compared among students from different years, and with results published in the literature. The main factors considered by the students in this decision were: broader or specific knowledge, contact with the patient, place of professional activity, and lifestyle after medical residency. For the women, the factors day-to-day stress of the specialty, the long-term relationship with the patient, and the ease of finding a job were significantly more relevant in the choice of specialty than for the men. Parents who were doctors also influenced the choice of specialty. The student's financial profile influenced the choice when related to long-term financial returns, being more relevant for students with monthly family incomes at the higher or lower ends of spectrum, i.e., - between 6 and 15 thousand Brazilian reals, or more than 20 thousand Brazilian reals. The specialty General Surgery was the first choice, predominantly among the male students, followed by Pediatrics, Clinical Medicine and Psychiatry. Conclusions: Lifestyle, place of professional activity and early financial returns were considered equally important by students across all the years evaluated. Due to the large number of variables evaluated, and the many factors that influence this decision, additional studies are needed, in order to make more significant comparisons with the literature.
\end{abstract}

Recebido em: 8/8/18

Aceito em: 17/12/18

\section{INTRODUÇÃO}

A escolha da especialidade médica é de suma importância para o estudante de Medicina, uma vez que representa a escolha da sua prática diária. Embora realização acadêmica, considerações financeiras e preferências de estilo de vida influenciem a escolha, os aspectos intrapessoais têm impacto na decisão do médico ${ }^{1}$.

Estilo de vida, personalidade, interesses e valores pessoais têm sido citados como importantes nesta decisão, mas nenhuma especialidade pode ser caracterizada por um único padrão de fatores ${ }^{2}$. Prestígio social, personalidade, gênero, recompensa financeira e estado civil também são citados como fatores de influência nesta escolha ${ }^{3}$.

A Comissão Mista de Especialidades (CME), composta por representantes da Comissão Nacional de Residência Médica (CNRM), da Associação Médica Brasileira (AMB) e do Conselho Federal de Medicina (CFM), reconhece 55 especialidades médicas, que devem contar com no mínimo dois anos de formação, e 59 áreas de atuação, com no mínimo um ano de formação ${ }^{4,5}$.
Testes psicométricos têm demonstrado grande confiabilidade e consistência na escolha da especialidade por parte dos estudantes de Medicina. Os testes podem ser importantes para pesquisadores, orientadores e estudantes, além de administradores que desejem identificar estudantes interessados em atenção primária mais cedo no treinamento médico ${ }^{6}$.

Na Europa e EUA, o tema tem sido abordado de maneira ampla desde a segunda metade do século XX. O material explicativo Roadmap to Choosing a Medical Specialty, da Stanford School of Medicine Academic, de 2015, propõe um algoritmo para a escolha da carreira médica conforme as preferências do estudante por cuidado direto ou indireto ao paciente ${ }^{7}$.

Utilizando o livro How to Choose a Medical Specialty, de Anita Taylor, a School of Medicine - University of Virginia desenvolveu um questionário online com 130 questões que compara as preferências dos estudantes às preferências dos profissionais de cada área. Para cada questão, pede-se que o estudante classifique a afirmação utilizando uma escala de 1 a 5 para a afirmação "mais verdadeira" ou "menos verdadeira". No final, é fornecido um ranking de especialidades, que se inicia por 
aquela com a qual o estudante mais se identifica e continua de forma decrescente em relação à interpretação do questionário.

No Brasil, o número de estudos nesta área é limitado. Poucas publicações mostram referências nacionais com instrumentos validados para aplicação de testes de aptidões e auxiliares na escolha da especialidade médica, evidenciando a necessidade de mais pesquisas nessa área. Em 2017, Caires et al. ${ }^{8}$ demonstraram consistência satisfatória e boa reprodutibilidade na tradução para o português, adaptação para a realidade brasileira e validação do Sci59, instrumento psicométrico de auxílio à escolha da especialidade médica, desenvolvido no Reino Unido.

\section{OBJETIVOS}

O presente estudo tem como objetivos avaliar os fatores que influenciam o acadêmico de Medicina na escolha da especialidade médica e identificar as áreas de atuação preferidas pelos estudantes dos quatro primeiros anos da faculdade para o exercício profissional do médico.

\section{MATERIAL E MÉTODO}

A pesquisa foi realizada com os acadêmicos do curso de Medicina, maiores de 18 anos, devidamente matriculados nos oito primeiros períodos do curso, numa instituição de ensino superior de Curitiba, no Paraná.

Foram incluídos todos os acadêmicos que aceitaram participar espontaneamente do estudo. Os estudantes responderam a um questionário elaborado e adaptado pelas autoras com base nas perguntas mais utilizadas em outros artigos publicados, contendo itens que os estudantes classificaram por uma escala Likert com pontuação de 1 a 5 , sendo 1 para discordo completamente e 5 para concordo completamente.

Os instrumentos de pesquisa foram aplicados entre os dias 14 e 18 de maio de 2018, e os dados coletados foram divididos em grupos conforme o período da graduação e sexo para realização das análises.

O estudo utilizou o método exploratório-descritivo com abordagem quantitativa. Os dados foram coletados e armazenados numa planilha do Microsoft Excel. A análise de dados foi realizada com auxílio do programa computacional SPSS v.22.0.

Os resultados são expressos por frequências e percentuais (variáveis qualitativas). A análise inferencial foi realizada por meio dos testes estatísticos Mann Whitney e Kruskal Wallis, sendo que valores de $\mathrm{p}$ menores que 0,05 foram considerados significativos.

O projeto de pesquisa foi aprovado pelo Comitê de Ética da instituição: Projeto $n^{\circ} 84171918.8 .0000 .5580$. Entrada no CEP: 19/04/2018. Aprovação: 27/04/2018.

\section{RESULTADOS}

A instituição possui 397 estudantes matriculados nos quatro primeiros anos do curso de Medicina, antes da fase do internato. Destes, 307 (78,3\%) estudantes aceitaram participar espontaneamente do trabalho, porém cinco foram excluídos das análises por não terem 18 anos completos, e outros 11 por não terem assinalado nenhuma das opções para sexo, período e idade. Dos 291 questionários válidos, 87 estudantes se identificaram como do sexo masculino e 204 como do sexo feminino. A distribuição dos estudantes por períodos está apresentada na Tabela 1.

\begin{tabular}{|cc|}
\hline \multicolumn{2}{c|}{ TABELA 1} \\
\hline Distribuição dos estudantes por períodos \\
\hline Período & Frequência $(\%)$ \\
\hline $1^{\circ}$ & $30(10,3 \%)$ \\
\hline $2^{\circ}$ & $37(12,7 \%)$ \\
\hline $3^{\circ}$ & $38(13,1 \%)$ \\
\hline $4^{\circ}$ & $39(13,4 \%)$ \\
\hline $5^{\circ}$ & $28(9,6 \%)$ \\
\hline $6^{\circ}$ & $43(14,8 \%)$ \\
\hline $7^{o}$ & $34(11,7 \%)$ \\
\hline $8^{\circ}$ & $42(14,4 \%)$ \\
\hline$T^{\circ}$ & 291 \\
\hline
\end{tabular}

A distribuição por faixa etária foi de 235 estudantes entre $18-24 \operatorname{anos}(80,7 \%), 45$ entre $25-30$ anos $(15,5 \%)$ e 11 com mais de 30 anos (3,8\%). De 286 respostas válidas, 25 (8,7\%) estudantes disseram ter pai ou mãe médicos.

Os dados sobre a renda familiar foram respondidos por $272(94,8 \%)$ estudantes, sendo $59(21,4 \%)$ com renda familiar maior que 20 mil reais por mês; 39 (14,1\%) com renda entre 15 e 20 mil reais; 45 (16,3\%) com renda entre 10 e 15 mil reais; $71(25,7 \%)$ entre 6 e 10 mil reais; e 62 (22,5\%) acadêmicos com renda familiar mensal inferior a 5 mil reais.

Sobre as especialidades preferidas pelos estudantes, obtivemos 288 respostas válidas. A mais citada foi Cirurgia Geral, sendo apontada por 35 acadêmicos como primeira escolha, seguida por Pediatria (28 acadêmicos) e Psiquiatria, juntamente com Clínica Médica (20 estudantes), conforme demonstrado na Tabela 2. Apesar de a Cirurgia Geral ter sido a especialidade mais citada, as especialidades das áreas clínicas foram preferidas por 167 estudantes (58\%), enquanto as cirúrgicas por $84(29,2 \%)$ e mistas por $37(12,8 \%)$ (Tabela 3$)$. Foram consideradas mistas as seguintes especialidades: Ginecologia e Obstetrícia, Oftalmologia, Ortopedia e Traumatologia, Otorrinolaringologia, Urologia. 


\begin{tabular}{lc}
\multicolumn{2}{c}{ TA BELA 2 } \\
Especialidade & \\
\hline Cirurgia Geral & Frequência (\%) \\
Pediatria & $35(12,1 \%)$ \\
Clínica Médica & $28(9,7 \%)$ \\
Psiquiatria & $20(6,9 \%)$ \\
Ginecologia e Obstetrícia & $20(6,9 \%)$ \\
Medicina de Família e Comunidade & $17(5,9 \%)$ \\
Dermatologia & $14(4,8 \%)$ \\
Cirurgia Plástica & $13(4,5 \%)$ \\
Endocrinologia & $11(3,8 \%)$ \\
Neurologia & $11(3,8 \%)$ \\
Outras & $11(3,8 \%)$ \\
Total & $108(37,5 \%)$
\end{tabular}

\begin{tabular}{|c|c|c|c|}
\hline & $\begin{array}{r}\text { ТАВ } \\
\text { cia por } \\
\text { a } x \text { cirú }\end{array}$ & $\begin{array}{l}\text { or períod } \\
\text { x mista) }\end{array}$ & \\
\hline Período & Clínica & Cirúrgica & Mista \\
\hline $1^{\mathrm{o}}$ & 23 & 4 & 2 \\
\hline $2^{\circ}$ & 16 & 16 & 5 \\
\hline $3^{\circ}$ & 21 & 11 & 6 \\
\hline $4^{\circ}$ & 25 & 7 & 7 \\
\hline $5^{\circ}$ & 14 & 9 & 5 \\
\hline $6^{\circ}$ & 21 & 18 & 3 \\
\hline $7^{\circ}$ & 18 & 7 & 8 \\
\hline $8^{\circ}$ & 28 & 12 & 1 \\
\hline
\end{tabular}

Os fatores considerados de maior influência na escolha da especialidade (pontuação média $>3,5$ ) foram: conhecimento mais amplo ou específico, contato com o paciente, local de atuação, estilo de vida após a residência, tempo para se dedicar à família ou lazer, relação a longo prazo com o paciente e grau de complexidade da especialidade. A Tabela 4 apresenta os 15 fatores que obtiveram maior pontuação média.

Quando correlacionados os fatores de influência na escolha da especialidade e ano letivo em curso, horas de trabalho e pressão no dia a dia da residência foram mais considerados pelos estudantes do quarto ano em relação aos demais.

Estilo de vida, local de atuação e retorno financeiro precoce foram considerados igualmente importantes por estudantes de todos os anos avaliados. Para estudantes do primeiro ano, tempo para se dedicar à família ou lazer foi menos considerado que para os demais anos. Os demais resultados estão descritos na Tabela 5 .

\section{TABELA 4}

Pontuação média da influência dos fatores na escolha da especialidade médica

Fatores Pontuação Média

Conhecimento mais amplo ou específico 4,1

Contato com o paciente 4,0

Local de atuação

Estilo de vida da especialidade após a residência

Tempo para se dedicar à família ou lazer

Relação a longo prazo com o paciente

Grau de complexidade da especialidade

3,7

3,6

Possibilidade de clínica e cirurgia

Facilidade em oportunidade de emprego

Interação com médicos de outras especialidades

3,5

Admiração por um docente

3,5

Retorno financeiro a longo prazo

Horas de trabalho

Estilo de vida durante a residência

Pressão do dia a dia no exercício da especialidade

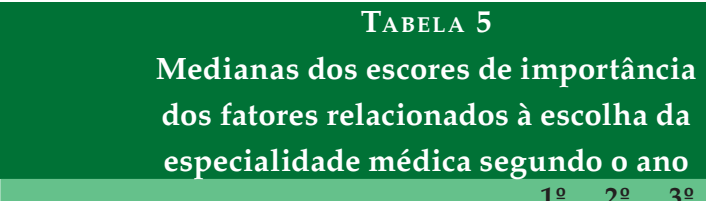

Fatores $\begin{array}{ccccc}1^{\circ} & 2^{\circ} & 3^{\circ} & 4^{\circ} \\ \text { Ano Ano Ano Ano } & p\end{array}$

Horas de trabalho $\begin{array}{lllll}3 & 3 & 3 & 4 & 0,000\end{array}$

Tempo para se dedicar à família ou lazer $\begin{array}{llllll}3 & 4 & 4 & 4 & \mathbf{0 , 0 0 0}\end{array}$ Pressão do dia a dia na residência $\quad \begin{array}{llllll}3 & 3 & 3 & 4 & \mathbf{0 , 0 1 0}\end{array}$

Pressão do dia a dia no exercício da especialidade

$\begin{array}{lllll}3 & 4 & 3 & 4 & \mathbf{0 , 0 1 4}\end{array}$

Estilo de vida da especialidade após a residência

Local de atuação profissional

Retorno financeiro precoce

Possibilidade de clínica e cirurgia

Influência de amigos

Influência de professores

Duração da residência

Contato com o paciente

Especialidade com maior status/prestígio social

Estilo de vida durante a residência

Retorno financeiro a longo prazo

Foco em emergência

Facilidade em oportunidade de emprego

Conhecimento mais amplo ou específico

Grau de complexidade da especialidade

Relação a longo prazo com o paciente

Dificuldade para entrar na residência

Foco em saúde pública

Interação com médicos de outras

especialidades

Interesse em pesquisa

Admiração por um mentor

Admiração por um docente

Influência de parentes

Influência de outros médicos

Distribuição por sexo da especialidade

\begin{tabular}{lllll}
4 & 4 & 4 & 4 & 0,014 \\
4 & 4 & 4 & 4 & $\mathbf{0 , 0 2 0}$ \\
3 & 3 & 3 & 3 & $\mathbf{0 , 0 3 1}$ \\
4 & 4 & 4 & 3 & $\mathbf{0 , 0 4 1}$ \\
1 & 2 & 2 & 1 & 0,114 \\
2 & 3 & 3 & 3 & 0,117 \\
2 & 3 & 3 & 3 & 0,128 \\
4 & 4 & 4 & 5 & 0,149 \\
\hline 2 & 2 & 3 & 2 & 0,193 \\
\hline 3 & 4 & 3 & 4 & 0,222 \\
3 & 4 & 4 & 4 & 0,267 \\
3 & 3 & 3 & 3 & 0,294 \\
4 & 4 & 4 & 4 & 0,432 \\
\hline 4 & 4 & 4 & 4 & 0,443 \\
4 & 4 & 4 & 4 & 0,456 \\
4 & 4 & 4 & 4 & 0,461 \\
\hline 2 & 2 & 3 & 3 & 0,539 \\
3 & 3 & 3 & 3 & 0,633 \\
4 & 4 & 3 & 4 & 0,633 \\
\hline 3 & 3 & 3 & 3 & 0,655 \\
\hline 3 & 4 & 3 & 3 & 0,699 \\
4 & 4 & 4 & 4 & 0,739 \\
1 & 2 & 1 & 1 & 0,892 \\
3 & 3 & 3 & 3 & 0,925 \\
1 & 1 & 1 & 1 & 0,932
\end{tabular}


Quando comparadas as medianas de cada um dos fatores entre os estudantes que se identificaram como do sexo feminino ou do sexo masculino, os que tiveram significância estatística na comparação entre os sexos foram: contato com o paciente, pressão do dia a dia no exercício da especialidade, relação a longo prazo com o paciente, facilidade em oportunidade de emprego, distribuição por sexo da especialidade, local de atuação profissional e foco em saúde pública. Destes, pressão do dia a dia no exercício da especialidade, relação a longo prazo com o paciente e facilidade em oportunidade de emprego foram mais considerados pelas mulheres do que pelos homens. A Tabela 6 apresenta todos os fatores e suas medianas distribuídas entre os sexos.

\begin{tabular}{|c|c|c|c|}
\hline $\begin{array}{c}\text { TABELA } 6 \\
\text { Mediana dos escores de import } \\
\text { dos fatores relacionados à escol } \\
\text { especialidade médica segundo }\end{array}$ & $\begin{array}{l}\text { ância } \\
\text { ha da } \\
\text { sexo }\end{array}$ & & \\
\hline Fatores & Fem. & Masc. & $\mathrm{p}$ \\
\hline Contato com o paciente & 4 & 4 & 0,001 \\
\hline $\begin{array}{l}\text { Pressão do dia a dia no exercício da } \\
\text { especialidade }\end{array}$ & 4 & 3 & 0,004 \\
\hline Relação a longo prazo com o paciente & 4 & 3 & 0,004 \\
\hline Facilidade em oportunidade de emprego & 4 & 3 & 0,009 \\
\hline Distribuição por sexo da especialidade & 1 & 1 & 0,015 \\
\hline Foco em saúde pública & 3 & 3 & 0,019 \\
\hline Local de atuação profissional & 4 & 4 & 0,036 \\
\hline Estilo de vida durante a residência & 4 & 3 & 0,063 \\
\hline Estilo de vida da especialidade após a residência & 4 & 4 & 0,070 \\
\hline Influência de professores & 3 & 2 & 0,071 \\
\hline Horas de trabalho & 4 & 3 & 0,098 \\
\hline Tempo para se dedicar à família ou lazer & 4 & 4 & 0,122 \\
\hline Influência de outros médicos & 3 & 3 & 0,243 \\
\hline Pressão do dia a dia na residência & 3 & 3 & 0,246 \\
\hline Duração da residência & 3 & 2 & 0,250 \\
\hline Interação com médicos de outras especialidades & 4 & 4 & 0,294 \\
\hline Retorno financeiro a longo prazo & 4 & 4 & 0,342 \\
\hline Especialidade com maior status / prestígio social & 2 & 2 & 0,350 \\
\hline Dificuldade para entrar na residência & 3 & 2 & 0,373 \\
\hline Interesse em pesquisa & 3 & 3 & 0,487 \\
\hline Influência de amigos & 1 & 2 & 0,495 \\
\hline Influência de parentes & 1 & 1 & 0,544 \\
\hline Possibilidade de clínica e cirurgia & 4 & 4 & 0,548 \\
\hline Retorno financeiro precoce & 3 & 3 & 0,609 \\
\hline Admiração por um mentor & 3 & 3 & 0,654 \\
\hline Conhecimento mais amplo ou específico & 4 & 4 & 0,665 \\
\hline Foco em emergência & 3 & 3 & 0,710 \\
\hline Admiração por um docente & 4 & 4 & 0,749 \\
\hline Grau de complexidade da especialidade & 4 & 4 & 0,999 \\
\hline
\end{tabular}

Foram comparadas as medianas dos fatores relacionados ao retorno financeiro precoce e a longo prazo com a renda familiar dos acadêmicos. Houve significância estatística apenas para retorno a longo prazo, sendo esta mais relevante para os estudantes com renda de 6 a 10 mil e de 10 a 15 mil reais e acima de 20 mil reais.

\begin{tabular}{|c|c|c|c|c|c|c|}
\hline $\begin{array}{r}\text { Mediana do } \\
\text { relacionad } \\
\text { a longo }\end{array}$ & $\begin{array}{r}\text { T } \\
\text { escores } \\
\text { s ao ret } \\
\text { razo se }\end{array}$ & $\begin{array}{l}\text { ABELA } \\
\text { de im } \\
\text { orno fi } \\
\text { gundo }\end{array}$ & $\begin{array}{l}7 \\
\text { portânc } \\
\text { inancei } \\
\text { a rend. }\end{array}$ & $\begin{array}{l}\text { cia dos } \\
\text { ro prec } \\
\text { a famil }\end{array}$ & $\begin{array}{l}\text { fatores } \\
\text { oce e } \\
\text { iar }\end{array}$ & \\
\hline & $\begin{array}{c}\text { Até R\$ } \\
5 \mathrm{mil}\end{array}$ & $\begin{array}{c}\text { De R\$ } \\
6 \text { a } 10 \\
\text { mil }\end{array}$ & $\begin{array}{c}\text { De R\$ } \\
10 \text { a } 15 \\
\text { mil }\end{array}$ & $\begin{array}{c}\text { De R\$ } \\
15 \text { a } 20 \\
\text { mil }\end{array}$ & $\begin{array}{c}\text { Maior } \\
\text { que } 20 \\
\text { mil }\end{array}$ & p \\
\hline $\begin{array}{l}\text { Retorno financeiro } \\
\text { precoce }\end{array}$ & 3 & 3 & 3 & 3 & 3 & 0,401 \\
\hline $\begin{array}{l}\text { Retorno financeiro a } \\
\text { longo prazo }\end{array}$ & 3 & 4 & 4 & 3 & 4 & 0,048 \\
\hline
\end{tabular}

Por fim, foram comparados os fatores influência de parentes e admiração por um mentor entre os acadêmicos que tinham ou não pai ou mãe médicos. Destes, ambos tiveram relevância estatística $(\mathrm{p}<0,05)$ (Tabela 8$)$.

\begin{tabular}{|c|c|c|c|}
\hline \multicolumn{4}{|c|}{$\begin{array}{l}\text { Mediana dos escores de importância dos } \\
\text { fatores relacionados à admiração por um } \\
\text { mentor e influência de parentes }\end{array}$} \\
\hline Fatores & Não & Sim & $\mathrm{p}$ \\
\hline Admiração por um mentor & 3 & 4 & 0,041 \\
\hline Influência de parentes & 1 & 3 & 0,000 \\
\hline
\end{tabular}

\section{DISCUSSÃO}

No Brasil, foi encontrado um dado inédito e paradoxal na formação de especialistas: cerca de $40 \%$ das vagas autorizadas de residência médica não chegam a ser ocupadas. Enquanto os residentes em cursos ou vagas preenchidas são 35.178, o total de vagas autorizadas chega a 58.077. São 22.899 vagas não ocupadas, o equivalente a $39,4 \%$ do total das autorizadas pela CNRM. Das 22.432 vagas autorizadas de R1 em 2017, foram de fato ocupadas 16.499, ou seja, uma diferença de 5.933 vagas ociosas. O mesmo estudo evidencia que os médicos residentes estão distribuídos de forma desigual no território nacional. A Região Sudeste tem 58,5\% dos 35.178 residentes inscritos em 2017 em todos os programas, seguida pelas regiões Sul (5.631 residentes, equivalentes a $16 \%)$, Nordeste $(14,2 \%)$, Centro-Oeste $(7,2 \%)$ e Norte $(4,1 \%)^{9}$.

Sabe-se que os acadêmicos de Medicina demonstram grande ansiedade em relação ao futuro e à escolha da especia- 
lização médica desde o início da graduação. Há uma pressão social, de colegas, familiares e do próprio aluno para escolher precocemente a especialidade que vai seguir.

Em estudo sobre a escolha da residência de Cirurgia Geral, observou-se que os homens são mais prevalentes nessa área ${ }^{10}$. Além disso, entre os acadêmicos que optam pela carreira cirúrgica, 30\% já têm essa decisão tomada antes mesmo do início do curso, diferentemente dos que escolhem a Clínica Médica, que optam de maneira progressiva, de acordo com o tempo, principalmente nos últimos dois anos de internato ${ }^{11}$. No presente estudo, 35 estudantes escolheram Cirurgia Geral como especialidade, sendo 22 do sexo feminino $(10,8 \%$ do total de mulheres) e 13 do sexo masculino (15\% do total de homens), demonstrando a existência de predomínio relativo masculino.

Na instituição do estudo, 9,7\% dos estudantes optaram pela Pediatria, especialidade que ficou na segunda posição de preferência. Embora a faculdade esteja vinculada a um grande hospital exclusivamente pediátrico do País, este fato parece não influenciar a escolha, já que nos dados demográficos nacionais a Pediatria aparece em primeiro lugar na preferência de recém-formados em Medicina9.

Estudos apontam que acadêmicos que se voltam para especialidades da área de atenção primária sofrem menos influência da percepção do prestígio que irão receber por sua escolha do que acadêmicos que se voltam para especialidades que não são da área de atenção primária ${ }^{12}$. Além disso, observa-se que acadêmicos mantêm uma visão de que na atenção primária irão receber menor remuneração do que em outras especialidades médicas, o que pode vir a ser um aspecto motivador para a falta de interesse pela Medicina de Família e Comunidade $^{13}$. Apesar disso, no presente trabalho a especialização de Medicina de Família e Comunidade ficou em sexto lugar na preferência dos estudantes dos quatro primeiros anos do curso, representando $4,8 \%$ dos estudantes e assumindo posição acima de especialidades como Dermatologia e Cirurgia Plástica. Este achado pode ser explicado pelo perfil e metodologia adotados pela faculdade e pelo fato de o retorno financeiro tardio não ter aparecido como fator importante na decisão dos estudantes de maneira geral.

Avaliando os dados demográficos de 2017, publicados em Demografia Médica no Brasil de 2018, a Medicina de Família e Comunidade é a primeira opção para apenas 1,5\% dos recém-formados, ocupando o décimo quinto lugar no ranking de especialidades preferidas.

Mundialmente, observa-se que a proporção de mulheres entre estudantes de Medicina aumentou. Continua controversa a forma como isso tem influenciado as mudanças observa- das nos padrões de escolha da especialidade médica, particularmente se esse crescimento da população feminina seria ou não responsável pela valorização do estilo de vida como determinante na escolha da carreira médica. Embora o estilo de vida tenha sido considerado um fator importante na escolha da especialidade pelas mulheres, não foi significativamente diferente da opinião dos homens.

No Brasil, o retorno financeiro e a qualidade de vida são apontados como principais motivações para a escolha da especialidade médica. Além disso, estilo de vida e oportunidade de emprego também se mostram aspectos relevantes. Esse mesmo estudo concluiu que o perfil financeiro do estudante não influencia a escolha da especialidade médica ${ }^{14}$. No entanto, o presente estudo demonstrou que o fator de retorno financeiro a longo prazo foi menos relevante para os acadêmicos com renda familiar abaixo de 5 mil reais do que para aqueles com renda acima de 20 mil reais.

Outros fatores, como afinidade com a especialidade, satisfação pessoal/profissional e gratificação, são determinantes para a escolha ${ }^{15}$. Em estudo realizado com acadêmicos de Medicina da Cesupa, os fatores que mais influenciaram a escolha da especialidade médica pelos alunos do primeiro ano foram afinidade pela área, influência dos pais e renda financeira esperada; no quarto ano, as principais influências foram também afinidade e renda financeira, seguidas da influência dos professores e do tempo livre esperado ${ }^{16}$. Em nosso estudo, não foi observada influência significativa dos professores e observou-se que a influência de parentes foi significativa apenas nos casos em que o pai ou a mãe são médicos.

Como o curso de Medicina da instituição tem apenas quatro anos de implantação, não foi possível avaliar os fatores que influenciam a decisão pela especialidade no período do ciclo do internato (dois últimos anos), o que provavelmente será feito em continuidade a este projeto.

\section{CONCLUSÃO}

Os principais fatores considerados pelos estudantes na escolha da especialidade foram: conhecimento mais amplo ou específico, contato com o paciente, local de atuação profissional e estilo de vida após a residência médica. Estilo de vida, local de atuação e retorno financeiro precoce foram considerados igualmente importantes por estudantes de todos os anos avaliados.

O perfil financeiro do estudante influencia a escolha quando se correlaciona a retorno financeiro a longo prazo, sendo mais relevante para estudantes com renda familiar entre 6-15 mil reais ou maior que 20 mil reais mensais. Pais médicos influenciam a decisão da escolha da especialidade. 
A Cirurgia Geral foi identificada como especialidade preferida entre os estudantes. A Pediatria ficou em segundo lugar no ranking geral das especialidades, seguida de Clínica Médica e Psiquiatria.

Devido à grande quantidade de variáveis avaliadas e de fatores que influenciam esta decisão, estudos adicionais e mais amplos são necessários para comparações mais significativas com a literatura disponível em nível mundial.

\section{REFERÊNCIAS}

1. Markert RJ, Rodenhauser P, El-Baghdadi MM, Juskaite K, Hillel AT, Maron BA. Personality as a Prognostic Factor for Specialty Choice: A Prospective Study of 4 Medical School Classes. Medscape J Med 2008; 27;10(2):49.

2. Borges NJ, Savickas ML. Personality and medical specialty choice: a literature review and integration. J CareerAssess. 2002;10:362-80.

3. Barshes NR, Vavra AK, Miller A, Brunicardi FC, Goss JA, Sweeney JF. General Surgery as a Career: A Contemporary Review of Factors Central to Medical Student Specialty Choice. J Am Coll Surg 2004;199(5):792-8.

4. Conselho Federal de Medicina. Resolução CFM no 1973/2011. Dispõe sobre a nova redação do Anexo II da Resolução CFM nº 1.845/08, que celebra o convênio de reconhecimento de especialidades médicas firmado entre o Conselho Federal de Medicina (CFM), a Associação Médica Brasileira (AMB) e a Comissão Nacional de Residência Médica (CNRM). Diário Oficial da União. Brasília, 1 ago. 2011; Seção 1, p. 144-7.

5. BRASIL. Lei nº 6,932, de 7 de julho de 1981. Dispõe sobre as atividades do médico residente e dá outras providências. Brasília, DF: Congresso Nacional, 1981.

6. Murdoch MM, Kressin N, Fortier L, Giuffre PA, Oswald L. Evaluating the Psychometric Properties of a Scale to Measure Medical Students' Career related Value. Acad Med 2001; 76(2):157-65.

7. Totman, A. Roadmap to Choosing a Medical Specialty. Stanford School of Medicine Academic Advising \& the Office of Medical Student Wellness; 2015.

8. Caires, IS, Goldberger, BU, Colares, MFA, Grant, J, Gale, R, Troncon, LEA. Tradução, Adaptação, Validação e Avaliação para Uso no Brasil de um Instrumento Britânico de Auxílio à Escolha da Especialidade Médica. Revbraseducmed 2017;41(3):379-389.

9. Scheffer, M, et al. Demografia Médica no Brasil 2018. São Paulo, SP: FMUSP, CFM, Cremesp, 2018.
10. Purim, KSM, Borges, LMC, Possebom, AC. Perfil do médico recém-formado no sul do Brasil e sua inserção profissional. RevColBrasCir 2016;43(4):295-300.

11. Bellodi, PL. Clínica ou cirurgia - um estudo sobre razões da escolha da especialidade. São Paulo Med. J. 2004;122(3):81-6.

12. Rogers, LQ, Fincher, RM, Lewis, LA. Factors influencing medical students to choose primary care or non-primary care specialties. Academic Medicine 1990;65(9):47-48.

13. Morra, DJ, Regehr, G, Ginsburg, S. Medical Students, Money, and Career Selection: Students' Perception of Financial Factors and Remuneration in Family Medicine. Family Medicine2009;41(2):105-110.

14. Corsi, PR, Fernandes, EL, Intelizano, PM, Montagnini, CCB, Baracat, FI, Ribeiro, MCSA. Fatores que influenciam o aluno na escolha da especialidade médica. Revbraseducmed 2014;38(2):213-220.

15. Cruz JAS, Sandy, NS, Vannucchi, TR, Gouveia, EM, Passerotti, CC, Bruschini, H, Srougi, M. Fatores determinantes para a escolha da especialidade médica no Brasil. Revista de Medicina 2010;89(1):32-42.

16. Sousa, IQ, Silva, CP, Caldas, CAM. Especialidade médica: escolhas e influências. Revbraseducmed 2014;38(1):79-86.

\section{CONTRIBUIÇÃO DOS AUTORES}

As autoras Jessica Belei Martins e Fernanda Pombo Rodriguez realizaram a elaboração do projeto, revisão bibliográfica, elaboração do questionário, coleta e análise dos dados, escrita do artigo. A autora Izabel Cristina Meister Martins Coelho contribuiu na co-orientação e revisão final do artigo. A autora Elisângela de Mattos e Silva contribuiu na supervisão de todas as etapas, orientação e revisão do artigo. Todas leram e aprovaram a versão final do artigo, assim como autorizaram a submissão dele à Revista Brasileira de Educação Médica.

\section{CONFLITO DE INTERESSES}

Não há.

\section{ENDEREÇO PARA CORRESPONDÊNCIA}

Jessica Belei Martins

Av. Sete de Setembro, 2346 - apto 804

Centro - Curitiba

CEP 80060-070 - PR

Email: jessica.belei@hotmail.com unrestricted use, distribution, and reproduction in any medium, provided the original work is properly cited. 